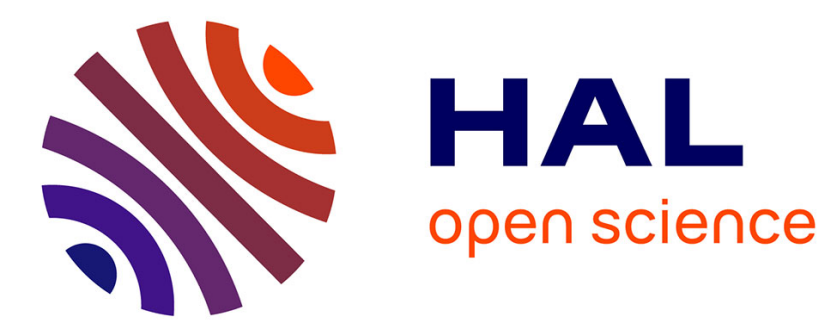

\title{
Sur les mesures électrostatiques (deuxième partie)
}

A. Cornu

\section{To cite this version:}

A. Cornu. Sur les mesures électrostatiques (deuxième partie). J. Phys. Theor. Appl., 1872, 1 (1), pp.87-98. 10.1051/jphystap:01872001008701 . jpa-00236812

\section{HAL Id: jpa-00236812 https://hal.science/jpa-00236812}

Submitted on 1 Jan 1872

HAL is a multi-disciplinary open access archive for the deposit and dissemination of scientific research documents, whether they are published or not. The documents may come from teaching and research institutions in France or abroad, or from public or private research centers.
L'archive ouverte pluridisciplinaire HAL, est destinée au dépôt et à la diffusion de documents scientifiques de niveau recherche, publiés ou non, émanant des établissements d'enseignement et de recherche français ou étrangers, des laboratoires publics ou privés. 
SUR LES MESURES ÉLEGTROSTATIQUES

(DECXIEME PARTIE);

PAR M. A. CORNU.

11. - Potentiel électrique d’un conducteur.

La notion du potentiel d'un conducteur électrisé s'est introduite naturellement dans l'étude des conditions d'équilibre électrique suivant les lois dícourcrtes par Coulomb. 
On remarque d'abord que, pour qu'une couche électrique reste cn équilibre à la surface d'un corps conducteur, il faut que la résultante de toutes les forces attractives et répulsives qu'clle exerce sur un point intérieur soit nulle. Cette condition est nécessaire, car, si elle n'était pas remplie, il y aurait, d'après les phénomènes connus, décomposition de l'électricité neutre du conducteur, attraction sur l'une des masses électriques, répulsion sur la masse de signe contraire; dès lors il y aurait mouvement de l'électricité, et l'équilibre ne serait pas établi. Le même raisonnement montre que cette condition est suffisante.

Quant à l'action réciproque des masses électriques réparties sur la surface du conducteur, il est évident qu'elle n'est pas nulle : il suffit, pour l'équilibre, que la résultante de toutes les forces soit en chaque point du conducteur répulsive et normale à l'élément de surface, c'est-à-dire dirigée dans la seule direction où le mouvement des masses électriques soit impossible; on prévoit mème, d'après un calcul très-simple dù à Coulomb, que la densité sur chaque élément de surface doit être proportionnelle à cette résultante $\left({ }^{1}\right)$.

( ${ }^{1}$ Cette résultante de toutes les masses electriques sur un élément de surface se calcule aisément. Divisons en deux parties l'action totale de la couche électrique sur un point très-voisin de la surface, celle de l'èlément que pour plus de simplicité on prendra circulaire, et celle du reste de la surface électrisée; le point attiré est supposé placé sur la normale élevée au centre de l'élément à une distance infiniment petite $e$, soit extérieurement, soit intérieurement. L'action de l'élément, qui, vu du point attiré, paraît un plan indéfini, reste évidemment la même en valeur absolue lorsque le point est d'un côté ou de l'autre, à l'intérieur ou à l'extérieur de la surface; toutefois elle change de signe. Mais, dans le premier cas, cette action doit faire équilibre à celle du reste de la surface; dans le second, elle la double, car on peut dire, à cause de la grande distance des points de cette surface, que le déplacement $2 e$ du point considéré est insensible.

L'action de l'élément, sur lequel la densité électrique moyenne est $\rho$, s'obtient un le décomposant en petites zones circulaires de rayons $\mathbf{R}, \mathbf{R}+d \mathbf{R}$, c'est-à-dire de surface $2 \pi \mathrm{R} d \mathrm{R}$ agissant à la distance $\sqrt{\mathrm{R}^{2}+e^{2}}$; la composante normale de l'action est, en supposant le point chargé de l'unité a'électricité,

$$
\rho \frac{2 \pi \mathbf{R} d \mathbf{R}}{\mathbf{R}^{2}+e^{2}} \frac{e}{\sqrt{\mathrm{R}^{2}+e^{a}}}=2 \pi \rho e \frac{\mathbf{R} d \mathbf{R}}{\left(\mathbf{R}^{2}+e^{2}\right)^{-1}}=\pi \rho \rho \frac{d}{d \mathbf{K}}\left(-\frac{1}{\sqrt{\mathbf{R}^{2}+e^{2}}}\right),
$$

dont l'intégrale prise depuis $R=0$ jusqu'a $\frac{R}{\rho}=\infty$ est $-2 \pi \rho$. Telle est laction de l'élément sur un point situé à une distance infiniment petite de la surface; on voit qu'elle est indépendante de $e$. L'action de la surface entière est zéro ou $\{\pi \%$, suivant que le point est intérieur ou extérieur. Il est bon de remarquer qu'on g'a pas, à pro- 
Analytiquement, la recherche de la distribution de l'électricité revient à répartir une masse électrique donnée en petites masses superficielles $m$, de telle sorte que, pour tout point intérieur situé à la distance $r$ de la masse $m$, la somme $\sum \frac{m}{r}$ étendue à toute la surface reste constante $\left({ }^{1}\right)$. C'est cette fonction, qui se ramène évi-

prement parler, déterminé la résultante de la surface sur l'élément, mais seulement sur un point infiniment voisin. Le calcul précédent ne s'appliquerait pas si l'on avait $e=0$. Néanmoins, le résultat est rigoureux en faisant abstraction de ce qui se passe dans l'intérieur de la couche électrique. La densité en chaque point de la surface est proportionnelle à la résultante des actions de la surface entière.

( ') Soient $m$ une masse quelconque positive ou négative et $x, y, z$ ses coordonnées satisfaisant à l'équation de la surface $\varphi(x, y, z)=0$; son action sur un point quelconque $a, b, c$, supposé chargé de l'unité d'électricité, aura pour composantes parallèles aux trois axes

$$
\frac{m}{r^{2}} \frac{x-a}{r}, \frac{m}{r^{2}} \frac{y-b}{r}, \frac{m}{r^{2}} \frac{z-c}{r} \text {. }
$$

La résultante totale aura pour composantes $X, Y, Z$ la somme de ces composantes élémentaires, somme étendue à toutes les masses de la surface,

$$
\mathbf{X}=\sum \frac{m}{r^{2}} \frac{x-a}{r}, \quad \mathbf{Y}=\sum \frac{m}{r^{2}} \frac{y-b}{r}, \quad \mathbf{Z}=\sum \frac{m}{r^{2}} \frac{z-c}{r} .
$$

La sommation doit être effectuée en donnant aux quantités $x, y, z$ les valeurs des coordonnées qui correspondent à la masse $m$, les lettres $a, b, c$ étant des constantes. Le résultat final ne contiendra que ces coordonnées $a, b, c$ et les paramètres qui servent à définir géométriquement la surface. De quelque manière que les quantités $a, b, c$ soient enveloppées dans le résultat final, on peut toujours faire par rapport à elles des opérations absolument indépendantes de la sommation relative à $x, y, z$. En particulier, considérons la variation de la distance $r$ quand $a, b, c$ varient infiniment peu; on a

d'où

$$
r^{2}=(x-a)^{2}+(y-b)^{2}+(z-c)^{2},
$$

par suite,

$$
r d r=-(x-a) d a-(y-b) d b-(z-c) d c
$$

$$
\frac{d r}{d a}=-\frac{x-a}{r}, \frac{d r}{d b}=-\frac{y-b}{r}, \frac{d r}{d c}=-\frac{z-c}{r},
$$

en exprimant par la caractéristique $d$ la dérivée partielle par rapport à l'une des lettres $a, b, c$, les deux autres étant supposées constantes. Substituant les valeurs que représentent ces dérivées dans $\mathrm{X}, \mathrm{Y}, \mathrm{Z}$, on aura

ou bien

$$
\mathrm{X}=\sum\left(-\frac{m}{r^{2}} \frac{d r}{d a}\right), \quad \mathrm{Y}=\sum\left(-\frac{m}{r^{2}} \frac{d r}{d b}\right), \quad \mathrm{Z}=\sum\left(-\frac{m}{r^{2}} \frac{d r}{d c}\right),
$$

$$
\mathbf{X}=\sum m \frac{d\left(\frac{1}{r}\right)}{d a}, \quad \mathbf{Y}=\sum m \frac{d\left(\frac{1}{r}\right)}{d b}, \quad Z=\sum m \frac{d\left(\frac{1}{r}\right)}{d c} .
$$

Ces symboles représentent deux groupes d'opérations entièrement indépendantes, puisqu'elles se rapportent i deux systemes de variables (la sommation se rapportant 
demment au calcul d'une intégrale douhle quion nomms potentiel. Nous ne considérerons provisoirement que le polentiel intérieur, c'est-à-dire la valeur constante que prend cette fonction lorsqu'on choisit pour origine des distances $r$ un point intérieur.

La réduction du problème à la sommation de quotients $\frac{m}{r}$ démontre que, si une loi de distribution est possible, toute distribution semblable, c'est-à-dire formée de masses proportionnelles, l'est également. En effet, si cette somme $V$ est constante pour un point quelconque, elle le restera encore si l'on multiplie les masses $m$ par un coefficient constant $k$, mais elle sera elle-mème multipliée par $k$. On retrouve ainsi le principe expérimental de Coulomb, qu'on peut énoncer sous la forme suivante : La distribution relative de l'électricité est indépendante de la quantité totale; ou encore sous la

$x, y, z$, la diflérentiation à $a, b, c)$; on peut donc intervertir leur ordre. Si l'on com. mence par la sommation, il vient

$$
\mathrm{X}=\frac{d}{d a} \sum \frac{m}{r}, \quad \mathrm{Y}=\frac{d}{d b} \sum \frac{m}{r}, \quad \mathrm{z}=\frac{d}{d c} \sum \frac{m}{r} .
$$

Ces formules montrent que, pour obtenir les trois composantes de l'action de masses agissant sur un point $a, b, c$ chargé de l'unité d'électricité, il suffit de calculer la somme $\sum \frac{m}{r}, m$ représentant la masse électrique située à la distance $r$ du point donné, et de prendre les trois dérivées partielles de ces fonctions par rapport aux coordonnées $a, b, c$.

Cette action sera nulle si la fonction $v=\sum \frac{m}{r}$, qu'on nomme potentiel, est constante quelles que soient les valeurs de $a, b, c$.

On comprend aisément comment la sommation se réduit pour une conche électrique continue à une intégrale double. Le lecteur s'exercera utilement à démontrer par le calcul que pour tous les points intérieurs d'une sphère uniformément électrisée le potentiel est constant; de même pour l'ellipsö̈de, en supposant à la couche la loi d'épaisseur indiquée dans la première partie.

Le potentiel $\mathrm{V}$ est susceptible d'une interprétation mécanique importante : c'est la fonction qui représente le travail córespondant au déplacement du point attire. Pour le démontrer, il suffit d'écrire l'expression du travail effectué par le deplacement $d a, d b, d c$ du point considéré chargé de l'unité d'électricité. Cette expression $\mathbf{F} d r$, ou son équivalente

$$
\mathbf{X} d a+\mathbf{Y} d b+\mathbf{Z} d c
$$

est evidemment égale à $d \mathbf{V}$.

Il en résulte que, dans l'intérieur d'un corps électrisé, on peut déplacer une masse électrique sans dépenser de travail. Quant à la valeur du potentiel intérieur, elle est mécaniquement représentée par la quantité de travail positif ou négatif qu'il faudrait dépenser pour transporter à l'infini l'unité d'électricité prise dans l'intérieur ou sur la surface du conducteur. 
forme équivalente : Le potentıel électrique d'un conducteur électrisé est proportionnel à sa charge et à une constante qui ne dépend que de la forme du conducteur.

Unité de potentiel. - Le potentiel d'un conducteur de dimensions infinies est évidemment nul, car le facteur $\frac{1}{r}$ qui multiplie chaque masse superficielle est nul : c'est ce qui fait dire que la terre a un potentiel nul ou sensiblement nul, et tous les conducteurs en contact avec elle sont dans le mème cas. Dans les autres cas où le potentiel du corps électrisé n'est pas nul, il est exprimé par un nombre qui dépend des unités de longueur et d'électricité. D'après ce qui a été dit plus haut, on conçoit que le potentiel puisse être exprimé en unités absolues.

Exemple.-Le potentiel $\mathbf{V}$ d'une sphère uniformément électrisée est facile à déterminer, puisqu'on peut choisir le centre de la sphère comme le point par rapport auquel on effectue la sommation : on trouve évidemment $\mathbf{V}=\frac{\mathbf{M}}{\mathbf{R}}$, c'est-à-dire la masse électrique totale divisée par le rayon. Le potentiel d'une sphère de rayon $R=\mathrm{I}$ et chargée de l'unité d'électricité représente l'unité de potentiel.

Loi du partage de l'électricité. - La condition d'équilibre électrique exigeant que le potentiel d'un conducteur soit constant pour tous les points intérieurs s'étend immédiatement à un système quelconque de conducteurs : dans l'intérieur de chacun d'eux, le potentiel, c'est-à-dire la somme $\sum \frac{m}{r}$, doit être constant, en faisant aussi entrer dans cette somme les masses électriques de tous les conducteurs du système. Si les divers conducteurs sont en communication, l'électricité se partage entre eux de telle sorte que la valeur de leur potentiel doit être la mème. On peut donc dire que l'équilibre électrique d'un système de conducteurs électrisés mis en communication s'obtient par l'égalisation du potentiel $\left({ }^{1}\right)$.

Cette condition si simple ne laisse pas que d'être assez complexe dans l'application à des conducteurs dont la disposition relative est

(') On compare quelquefois le partage de l'électricité entre divers conducteurs au partage d'un liquide dans un système de vases communiquants. Le potentiel initial de chaque conducteur représente le niveau initial de chaque réservoir, la quantité d'électricité, la quantité de liquide. Cette quantité constante se partage de façon que le niveau final soit le mème dans tous les vases. 
variable, parce qu'à chaque nouvelle position l'influence réciproque varie et la distribution change à la surface de chacun d'eux ; d'où il résulte que le potenticl intéricur d'un conducteur, mème chargé d'une quantité fixe d'électricité, ne reste nullement constant lorsque des corps électrisés ou non se déplacent dans le voisinage. Mais, dans le cas où l'on suppose les conducteurs assez éloignés pour que leur influence mutuelle soit négligeable, on peut réduire la loi du partage de l'électricité entre ces conducteurs à une très-grande simplicité, en concevant la communication électrique établie par un fil dont la masse électrique n'aurait pas d'influence appréciable. Dans ces circonstances, on reconnait aisément la corrélation entre le potentiel et la température, et entre les problemes de calorimétrie et ceux de l'électrométrie.

Un corps conducteur étant donné, si on le charge de quantités croissantes d'électricité, son potentiel augmente dans le mème rapport.

La quantité d'électricité nécessaire pour faire acquérir à un conducteur l'unité de potentiel mesure la capacité électrique de ce conducteur.

La capacité d'un conducteur de forme donnée, réduit par similitude à l'unité de surface, se nomme capacilé spécifique de la forme donnée.

Un corps conducteur de la chaleur étant donné, si on le charge de quantités croissantes de chaleur, sa température augmente dans lo mème rapport.

La quantité de chaleur qui éève de l'unité la température d'un corps mesure la caparité calorifuque de ce corps.

La capacité calorifique d'un corps de nature donnée, réduit à l'unité de poids, se nomme chaleur spécifrque de la substance donnée.

L'équation d'équilibre électrique est la mème que celle de l'équilibre calorifique :

Deux conducteurs de capacités électriques $c, c^{\prime}$, chargés de quantités d'électricité $q, q^{\prime}$, étant mis en communication par un long fil, ont pour potentiel final $\mathrm{V}$, déduit de l'équation

$$
\left(\frac{q}{c}-V\right) c=\left(v-\frac{q^{\prime}}{c^{\prime}}\right) c^{\prime},
$$

leur potentiel initial étant respectivement

$$
\mathrm{V}=\frac{q}{c}, \quad \mathrm{~V}=\frac{q^{\prime}}{c^{\prime}} ;
$$

d'où l'on conclut

$$
\mathrm{V}=\frac{q+q^{\prime}}{c+c^{\prime}} .
$$

Deux corps de capacitéscalorifiques $c$, $c^{\prime}$, aux températures initiales $t, t^{\prime}$, étant mis en contact, arrivent à la température finale $\mathbf{T}$, déduite de l'équation

$$
(t-\mathrm{T}) c=\left(\mathbf{T}-t^{\prime}\right) \boldsymbol{c}^{\prime},
$$

les quantitis initiales de chaleur absorbées pour passer de la température zéro aux températures $t, t^{\prime}$ étant respectivement

$$
q=c^{t}, \quad q^{\prime}=c^{\prime} t^{\prime}
$$

on a d'ailleurs

$$
\mathrm{T}=\frac{c t+c^{\prime} t^{\prime}}{c+c^{\prime}}=\frac{q+q^{\prime}}{c+c^{\prime}} .
$$


Mesure expérimentale du potentiel. - La simplicité de l'expression du potentiel d'une sphère uniformément électrisée réduit la mesure absolue du potentiel d'un conducteur à une opération très-facile : il suffit d'établir la communication avec un fil entre re conducteur et une petite sphère de rayon $r$ servant de plan d'épreuve.

Soit $m$ la masse d'électricité emportée par la sphère (masse déterminée, comme on l'a vu précédemment, à l'aide de la balance de Coulomb); son potentiel est $r=\frac{m}{r}$ : c'est la valeur commune au conducteur et à la sphère au moment de la communication; soit $\mathbf{V}_{0}$ le potentiel primitif du conducteur et $c$ sa capacité électrique; le potentiel initial de la sphère étant zéro, sa capacité $c^{\prime}$ telle que $\frac{\mu}{r}=\mathrm{I}$, d'où $\mu=c^{\prime}=r$, l'équation d'équilibre donne alors

$$
\left(\mathrm{V}_{\mathrm{b}}-v\right) c=v r \quad \text { ou } \quad \mathrm{V}_{\mathrm{o}}=v\left(\mathrm{t}+\frac{r}{c}\right) .
$$

Si la capacité de la sphère est négligeable par rapport à celle du conducteur, on peut écrire $\mathbf{V}_{0}=\nu$, c'est-à-dire prendre pour potentiel du conducteur celui de la sphère. Si cette approximation ne suffisait pas, il faudrait déterminer ce rapport; à cet effet, on pourrait simplement réduire à zéro le potentiel de la sphère en la mettant en communication avec le sol; cn la remettant en communication lointaine avec le conducteur, on lui trouverait un noureau potentiel "' tel que

$$
v=v^{\prime}\left(1+\frac{r}{c}\right):
$$

d'où l'on conclurait

$$
V_{0}=\frac{v^{2}}{v^{\prime}} \text {. }
$$

La petite sphère conductrice joue donc le rôle d'un thermomètre électrique. Toutefois, il y a lieu de remarquer la dillérence des méthodes dans l'étude des deux agents, électricité et chaleur : d'un còté, la notion primitive est la température, on en a déduit celle de quantité de chaleur; de l'autre, c'est l'inverse, la notion de quantité d'électricité a servi de point de départ, et l'on s'est élevé à la notion de potentiel. Aussi, pour déterminer la température électrique, on fait l'analogue d'une opéraition de calorimétrie : on procède absolu- 
ment en électricité de la manière proposée, à tort ou à raison, pour déterminer la température d'un foyer, en plongeant dans un calorimètre un bloc de métal qui aurait séjourné dans le foyer pendant un temps assez long pour que l'équilibre de température fút établi $\left({ }^{1}\right)$.

Mesure de la capacité électrique d'un conducteur. - L'opération de la mesure du potentiel d'un conducteur sert en mème temps a déterminer sa capacité. Do l'équation

$$
v=v^{\prime}\left(1+\frac{r}{c}\right)
$$

on conclut

$$
c=\frac{r v}{v-v^{\prime}} .
$$

On remarquera toutefois que, pour atteindre une précision suffisante dans les mesures, il faut que la sphère de comparaison, au lieu d'être très-petite, soit de mème ordre de grandeur que le conducteur donné. Il sera donc bon de procéder cee la manière suivante: on partagera par communication lointaine l'électricité du conducteur avec une grande sphère à l'état naturel, ct, d'après l'indication d'une petite sphère scrvant de plan d'épreuve, on en conclura le potenticl $v$ de la grande sphère; on répétera une seconde fois la mème opération; le nouveau potentiel $v^{\prime}$ ot le rayon $r$ de cette sphère fourniront par substitution, dans la formule ci-dessus, la valeur de la capacité du conducteur. On reconnait dans ces opérations les mesures empruntées aux expériences de Weber et Kohlrausch citées dans la première partie de cet exposé.

Remarque. - Il est très-important de remarquer que la capacité électrique d'un conducteur ne reste fixe que dans le cas où ce conducteur n'est soumis à aucune influence extérieure : l'approche d'une masse conductrice change complétement la loi de distribution de la masse électrique primitive, par suite le potentiel intérieur et,

( $\left.{ }^{1}\right)$ Les unités usitées en chaleur ne sont pas absolues, car elles renferment deux definitions arbitraires : celle de la température et celle de la calorie. Toutelois, depuis la découverte de l'équivalence de la chaleur et du travail, l'une des définitions arbitraires peut être rejetéc. Il suffit de prendre pour unite de ehaleur la /36e partie de la caloric actuelle (une calorie equivatut a $\nmid 36$ hilogrammetres environ); les quantités de chaleur sont alors expimeen par le nombre de kilogrammetren squivalent. 
'n définitive, la capacité électrique. Ainsi l'ensemble de deux sphères égales qui se touchent n'a pas, comme capacité, le double de la rapacité de l'une d'elles. Les condensateurs en offrent un exemple encore plus frappant.

Électromètre Thomson. - La mesure relative des potentiels et surtout de très-petite's diflérences de potentiel existant entre divers conducteurs est un problème expérimental d'une grande importance, au double point de vue de la démonstration des lois précédentes et de l'identification des phénomènes produits par le courant des piles avec les plı́nomènes électrostatiques.

Ce problème a été résolu par M.William Thomson d'une manière très-simple et très-complète, à l'aide de son électromètre. Cet appareil se compose, comme pièces essentielles, d'une lame d'aluminium très-légère, suspendue horizontalement, comme l'aiguille d'une boussole, à un fil de torsion; elle est maintenue à une charge électrique assez forte et bien constante, et reste en équilibre entre deux paires de conducteurs qui l'enveloppent complétement. L'image qui représente le micux la forme de ces conducteurs est celle d'une boite circulaire, forme dite bonbonnière (fig. I), qui aurait été

Fiğ.

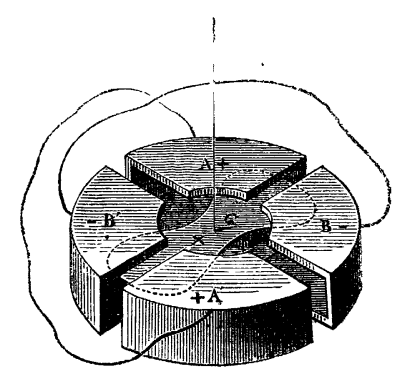

coupée en quatre suivant deux diamètres rectangulaires. On a ainsi quatre secteurs, $\mathrm{A}, \mathrm{B}, \mathrm{A}^{\prime}, \mathrm{B}^{\prime}$, qu'on rapproche de facon à reconstituer la boîte en laissant entre eux un petit intervalle. La fig. I représente en perspective ces secteurs. L'aiguille d'aluminium $\mathbf{G}$, qui a la forme d'un 8, est suspendue au centre de cette espèce de boîte et réglée par une torsion convenable, de façon que sa ligne médiane, dans sa position d'équilibre, soit parallèle à l'un 
des diametres de séparation des secteurs. I a charge constante que possède cette aiguille est maintenue par la communication permanente avec l'armature d'un condensateur par l'intermédiaire du fil de suspension. On donne cette charge en mettant en communication l'armature interne avec une source électrique par l'intermédiaire d'une petite pièce mobile.

Les quatre secteurs, étant parfaitement symétriques par construction, exercent sur l'aiguille des actions dont la résultante est nulle, lorsqu'ils ont tous quatre une charge égale ou nulle, c'est-à-dire lorsqu'ils ont le mème potentiel ou un potentiel nul. Mais, si l'on établit une dissymétrie dans leur charge, l'aiguille est déviée de sa position d'équilibre. On obtient le maximum d'eflet en faisant communiquer les secteurs deux a deux et en croix, de façon que chaque paire produise sur l'aiguille un couple de rotation. Il est facile de voir, en faisant une hypothèse quelconque sur les signes des variations de charge (ou de potentiel), que les deux couples tendent à produire une rotation de l'aiguille dans le mème sens.

Le fil de torsion est assez rigide pour que l'angle de déviation de l'aiguille soit très-petit, de sorte qu'on peut, sans crreur sensible, supposer, comme dans la boussole des tangentes, que la distance réciproque des divers points de l'aiguille aux secteurs n'a pas changé. Dans ces circonstances, on peut admettre que la capacité électrique des secteurs est invariable, et, par suite, que leur variation de potentiel est proportionnelle à la variation de la masse électrique qu'ils possèdent. Dès lors l'action exercée sur l'aiguille par chacun des couples de secteurs est, d'après la loi de Coulomb, proportionnelle au produit de la masse électrique de l'aiguille par la variation ou mieux la différence de leurs charges, ou, ce qui revient au mème, par leur différence de potentiel. Comme les déviations sont ellesmêmes proportionnelles au couple résultant de cette action totale, la différence de potentiel se trouve mesurée directement. On amplifie les faibles déviations de l'aiguille en observant sur l'échelle divisée l'image d'un point lumincux réfléchie sur un miroir concave excessivement léger, fixé convenablement à l'aiguille.

Cet appareil permet de vérifier toutes les lois indiquées plus haut sur les relations entre l'électricité et la chalcur. Il est précieux à deux points de vue : le premier, en ce qu'il permet de réaliser d'une manière rigourcuse la condition de communication lointaine asec 
un fil, et par suite de mesurer directement ( $\left.{ }^{1}\right)$ la différence de potenticl entre le sol, dont on suppose le potentiel nul, et le conducteur donné; le second avantage, c'est son excessive délicatesse, qui permet d'opérer avec des charges si faibles que la déperdition par l'air, les supports, les pointes, etc., est presque insensible.

D'ailleurs cette sensibilité peut être réglée à volonté, car elle est directement proportionnelle à la charge de l'aiguille. On arrive à une telle délicatesse, qu'on parvient à constater la charge produite par le plus léger frottement d'une étoffe sur un conducteur isolé, et, ce qui est plus remarquable encore, à mesurer la différence de potentiel permanente qui existe entre les deux pôles d'un couple voltaïque.

La fig. 2 donne une idée de l'ensemble de l'appareil. Les pièces essentielles décrites plus haut sont enfermées dans une cloche de

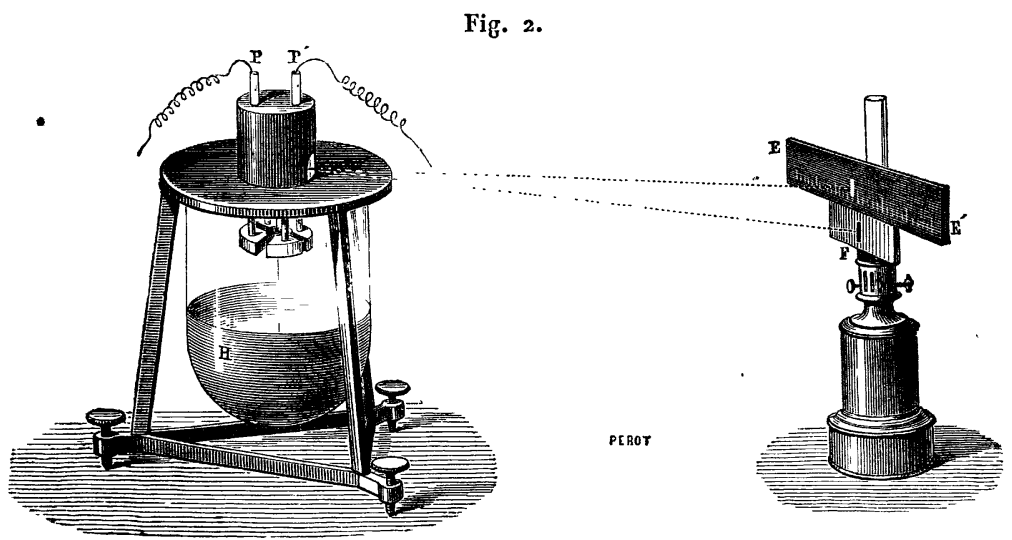

verre $H$, dont le fond hémisphérique est en bas. Cette cloche sert en même temps de condensateur, car elle est revêtue d'une feuille d'étain à l'extérieur et remplie à moitié d'acide sulfurique, qui joue à la fois le rôle de desséchant et celui de conducteur formant l'armature interne. Deux tiges, $\mathrm{P}, \mathrm{P}^{\prime}$, sortent au dehors et communiquent avec les deux paires de quadrants; une lampe éclaire une fente dont l'image conjuguée va se former sur l'échelle divisée $\mathrm{EE}^{\prime}$ :

(') En tenant compte, bien entendu, de la capacité propre des secteurs. I. 
le déplacement de cette image mesure avec une grande précision les déviations de l'aiguille.

Parmi les dispositifs très-ingénieux que contient cet électromètre, il faut citer : $\mathbf{r}^{\circ}$ un véritable petit électrophore à rotation (replenisher) destiné à réparer les pertes du condensateur, ou mieux à régler cette charge; $2^{\circ}$ un indicateur de la charge, formé par une petite balance de construction spéciale, qui permet de ramener toujours le condensateur à la même charge et, par suite, d'obtenir des indications comparables.

(A suivre.) 